\title{
Skin Metastasis in a patient with Small-Cell Lung Cancer
}

\author{
Kamonpun Ussavarungsi, MD, Minji Kim, BA, Lukman Tijani, MD
}

\begin{abstract}
A 64-year-old woman presented with abdominal pain and back pain. Physical examination revealed a subcutaneous nodule in the anterior abdominal wall. An ultrasoundguided biopsy revealed small cell lung carcinoma (SCLC), and additional workup revealed extensive stage small cell lung carcinoma with involvement of the left lung and the abdominal viscera. The patient underwent chemotherapy with a good initial response. Lung carcinoma infrequently metastasizes to the skin. However, these lesions, when present, have important prognostic implications since these patients typically respond poorly to chemotherapy and have a mean survival of about five months. Careful skin evaluation is essential in the evaluation of patients with possible bronchogenic carcinoma.
\end{abstract}

Key words: bronchogenic carcinoma, neoplasm metastasis, skin neoplasm, small cell lung cancer $(\mathrm{SCLC})$

\section{INTRODUCTION}

Cutaneous metastases of lung cancer are infrequent but have important diagnostic and prognostic value. Rarely, skin changes are the initial signs of underlying lung cancer, and common sites include the chest, back, abdomen, head, and neck. These metastases are usually single or multiple painless nodules that may be mobile or fixed. While the skin metastasis rate of large cell lung carcinoma is high, it is low for the squamous and small cell lung carcinomas. A definitive diagnosis can be made by a biopsy of the skin lesion with histological analysis. Lung cancer patients who present with cutaneous metastasis have a poor prognosis, with a mean survival of approximately five months. We present a rare case of cutaneous metastasis in a patient with small cell lung cancer.

Corresponding author: Kamonpun Ussavarungsi Contact Information: Ussavarungsi.Kamonpun@mayo.edu DOI: 10.12746/swrccc2013.0101.011

\section{Case Presenttion}

A 64-year-old woman with a past history of hypertension and tobacco abuse presented to the Emergency center with severe abdominal pain and back pain for two months. The abdominal pain was cramping and radiated to the back. There were no exacerbating or relieving factors. She denied fever, nausea, vomiting, diarrhea, chest pain, dyspnea, and cough. She had decreased appetite, fatigue, and generalized weakness but no weight loss.

On examination, vital signs included a temperature of $97.5^{\circ} \mathrm{F}$, a heart rate of 95 beats $/ \mathrm{min}$, a respiratory rate of 20 breaths/min, BP of $178 / 95 \mathrm{mmHg}$, and $\mathrm{O} 2$ saturation of $96 \%$ on room air. General examination showed a mildly confused middle-aged white female who was not in acute distress. She had no palpable lymphadenopathy. Her heart and lungs were within normal limits. The abdomen was soft with no tenderness. There was a nontender palpable subcutaneous mass approximately two $\mathrm{cm}$ in diameter in the left upper anterior abdominal wall.

Laboratory studies included hemoglobin level $13.8 \mathrm{~g} / \mathrm{dl}$, white blood cell count $12,500 / \mu \mathrm{L}$, potas- 
sium $3.7 \mathrm{mmol} / \mathrm{l}$, chloride $93 \mathrm{mmol} / \mathrm{l}$, bicarbonate 30 $\mathrm{mmol} / \mathrm{l}$, BUN $9 \mathrm{mg} / \mathrm{dl}$, creatinine $0.4 \mathrm{mg} / \mathrm{dl}$, calcium $9.4 \mathrm{mg} / \mathrm{dl}$, albumin $3.5 \mathrm{~g} / \mathrm{dl}$, AST 36 units $/ \mathrm{l}$, ALT 12 units/l, and alkaline phosphatase 85 units/l. Serum amylase was elevated at 135 units/l (25-100 units/L), lipase 427 units/l (10-52 units/L), CEA $31 \mathrm{ng} / \mathrm{ml}$ ( 0.2$3.4 \mathrm{ng} / \mathrm{ml}$ ), and CA 19-9 34.1 units $/ \mathrm{ml}$ ( 0.6-35 units/ $\mathrm{ml})$. Urinalysis was normal.

The patient's chest radiograph exhibited left hilar and perihilar densities (Figure 1). A CT scan of the chest showed a large left hilar and perihilar mass measuring $10 \mathrm{~cm}$ with encasement of the left main bronchus and right pulmonary artery and mediastinal extension with mediastinal lymphadenopathy (Figure 2). A CT of the abdomen and pelvis with and without contrast showed multiple hypodense lesions in the liver of approximately one $\mathrm{cm}$ in size, a solid mass in the pancreatic body, a lobulated anterior lower pole of the right kidney, bilateral adrenal nodules, and retroperitoneal lymphadenopathy. An ultrasound-guided biopsy was obtained from the subcutaneous mass in the left mid abdominal wall and revealed small cell carcinoma of the lung (Figures 3 and 4). A bone scan was negative. The patient was diagnosed with CT4N2M1b Extensive stage SCLC. Her abdominal pain was due to acute pancreatitis.

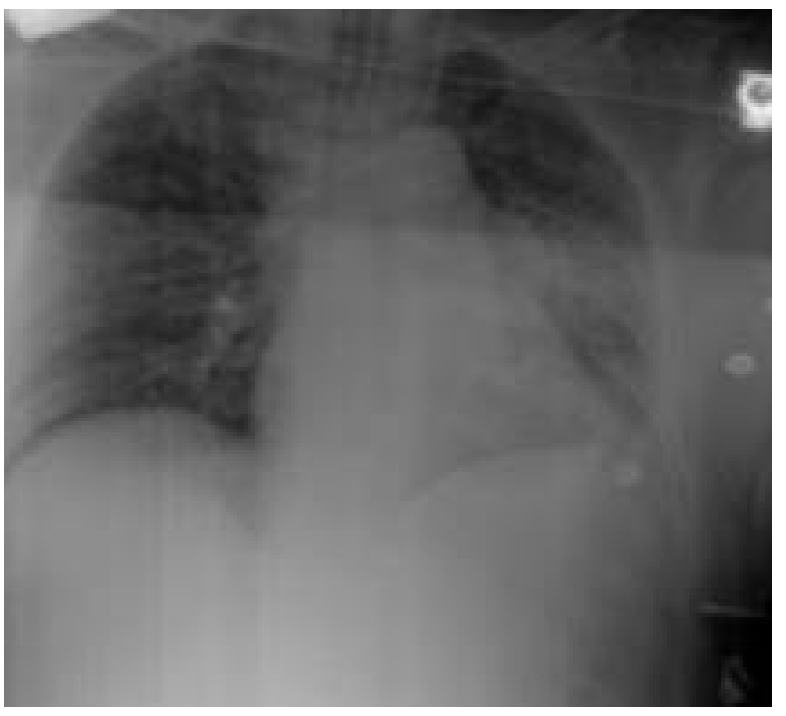

Figure 1. Portable chest radiograph revealed left hilar and perihilar density.

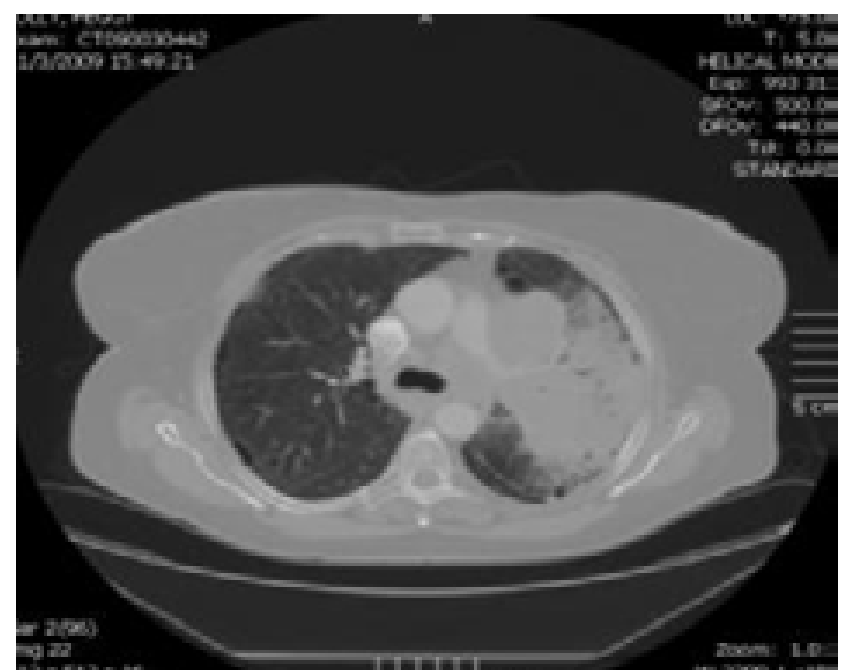

Figure 2. Large heterogeneous enhancing left hilar and perihilar mass measuring $10 \mathrm{~cm}$ with encasement of the left main bronchus and right pulmonary artery. Distal atelectasis and consolidation of the left upper and lower lobes. Enlarged confluent mediastinal lymph nodes.

A CT of her head with contrast revealed an eight $\mathrm{mm}$ mass in the left cerebral peduncle. Whole brain radiation and dexamethasone were started immediately with a marked improvement of her mental status.

She received four cycles of doublet chemotherapy with Platinum and tolerated it well. CT scans of the brain, chest, and abdomen were repeated during follow-up and revealed no evidence of brain metastasis, smaller left hilar nodes, a smaller left upper lobe pulmonary nodule, and minimal, if any, hepatic, renal, or adrenal disease. The subcutaneous nodule also resolved.

However, her disease progressed; she was started on IV topotecan, but could not tolerate it because of poor performance status, and was referred to hospice. 


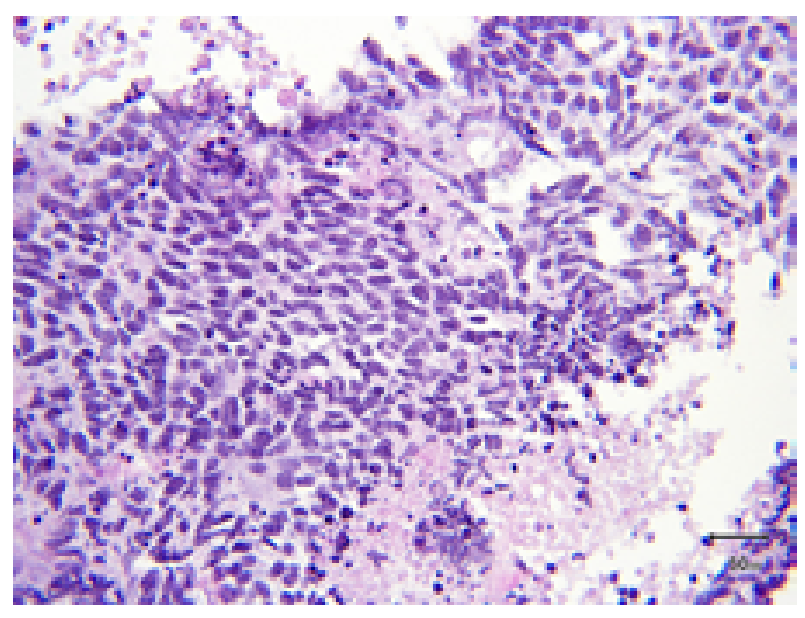

Figure 3. Subcutaneous mass tissue biopsy. Hematoxylin and eosin stain reveals black, variably sized nuclei within scant cytoplasm, molding and crush artifact.

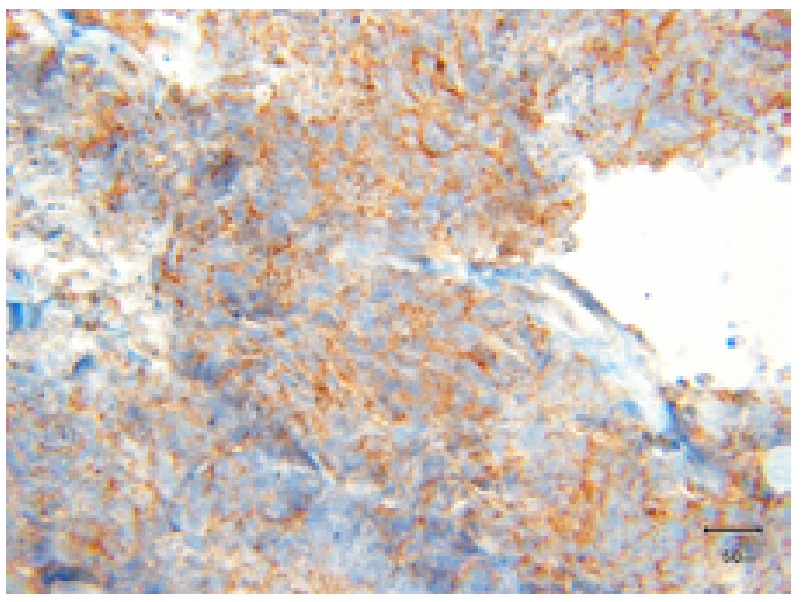

Figure 4. Immunohistochemistry with synaptophysin was positive.

\section{Discussion}

SCLC arises from bronchial epithelial cells and is related to the neuroendocrine Kulchitsky cells, a type of intestinal epithelial cells. SCLC is fatal and most patients die within one year of presentation, with median survival for extensive stage disease being 6-8 months. ${ }^{18}$ SCLC has a tendency to progress rapidly and to metastasize early and extensively. Common metastatic sites include lung, liver, bone, adrenal gland and central nervous system. ${ }^{19}$

Cutaneous metastases from the lung are not very common and indicate a poorer prognosis. The percentage of patients with lung cancer who develop skin metastasis ranges from $1-12 \%$. ${ }^{9}$ Between $7-24 \%$ of these patients have cutaneous lesions as an initial sign of malignancy. ${ }^{10}$ Patients often present with nodules which can be single or multiple and varied in size; less common lesions include papules, plaques, ulcerations, and sclerodermoid lesions. "11 Lung cancer with cutaneous metastasis usually involves the anterior chest, abdomen, head, neck, and back." Adenocarcinoma and large cell carcinoma have a higher incidence of cutaneous metastasis than squamous cell and small cell carcinoma. ${ }^{11}$ In addition, lung cancer in the upper lobes has a higher tendency to develop cutaneous metastasis. ${ }^{12}$ Patients with metastatic skin lesions usually have other internal visceral metastases and have a poor prognosis. Reported mean survival after skin metastasis is approximately five months. ${ }^{13}$ Patients who present with skin lesions earlier have a lower survival rate than patients who develop skin metastasis later in the disease course.

Various diagnostic and treatment modalities are involved in the next steps of patient management. Biopsy of skin lesions is an important component in making the diagnosis of lung cancer with cutaneous metastasis. The histological subtype can be diagnosed by biopsy, and imaging with chest radiographs and computed tomography is used to identify the primary site. Staging is done by PET/CT and MRI of the brain.

Treatment of solitary skin metastasis includes surgery combined with either or both chemotherapy and radiation; multiple cutaneous lesions may be better treated with chemotherapy. ${ }^{10}$ Patients with resectable skin lesions have better survival than those with multiple, non-resectable sites and are candidates for surgery.

When the metastases are multiple, chemotherapy is used as the initial treatment. For extensive stage SCLC, the combination of Etoposide and Cisplatin is the standard first-line chemotherapy regimen. However, to reduce toxicity, especially in patients with extensive disease who cannot tolerate cisplatin, carboplatin can be substituted for cisplatin. Kamble et al reported an initial reduction in nodule size when 
patients were treated with chemotherapy consisting of cisplatin, VP-16, cyclophosphamide, doxorubicin, and vincristine, but the skin lesions later progressed. ${ }^{17}$ Due to the aggressive nature of lung cancer with cutaneous metastasis, both chemotherapy and radiation therapy may be effective only as palliative treatment. ${ }^{17}$

\section{Key PoInts}

1. Skin metastasis occurs infrequently in patients with bronchogenic carcinoma.

2. These skin lesions have important diagnostic value and prognostic implications.

3. Some patients have partial responses to chemotherapy.

4. Radiation therapy can provide palliative care for pain and bleeding with skin metastasis.

Author Affiliation: Kamonpun Ussavarungsi is a fellow in pulmonary and critical care medicine at the Mayo Clinic Florida, 4500 San Pablo Road, Jacksonville, FL 32224. Minji Kim is a medical student at Texas Tech University Health Science Center in Lubbock, TX. Lukman Tijani is an oncologist in the Department of Internal Medicine at Texas Tech University Healh Science Center in Lubbock, TX.

Received: $11 / 15 / 2012$

Accepted: 01/31/2013

Reviewers: Kenneth Nugent, MD, Rishi Raj, MD

Published electronically: 02/12/2013

Conflict of Interest Disclosures: None
4. Barbagallo J, Tager P, Ingleton R, et al. Cutaneous tuberculosis: diagnosis and treatment. American Journal of Clinical Dermatology 2002; 3: 319-328.

5. Welsh O, Vera-Cabrera L, Rendon A, et al. Coccidioidomycosis. Clinics in Dermatology 2012; 30: 573-591.

6. Lopez-Martinez R, Mendez-Tovar L. Blastomycosis. Clinics in Dermatology 2012; 30: 565-572.

7. Lodha S, Sanchez M, Prystowsky S. Sarcoidosis of the skin, a review for the pulmonologist. Chest 2009; 139: 583-596.

8. Fernandez-Faith E, McDonnell J. Cutaneous sarcoidosis: differential diagnosis. Clinics in Dermatology 2007; 25: 276-287.

9. Mollet T, Garcia C. Skin metastasis from lung cancer. Dermatology Online Journal 2009.

10. Garrido M, Ponce GC, Martinez S, et.al. Cutaneous metastasis of lung cancer. Clinical \& Translational Oncology 2006; 8: 330-3.

11. Hidaka T, Ishii Y, Katmura S. Clinical features of skin metastasis from lung cancer. Internal Medicine 1996; 35: 459-462.

12. Coslett LM, Katlic MR. Lung cancer with skin metastasis. Chest 1990; 97: 757-9.

13. Terashima T, Kanaqawa M. Lung cancer with skin metastasis. Chest 1994; 106: 1448-50.

14. Garrido M, Rufete M, Ponce GC, et al. Skin metastases as first manifestation of lung cancer. Clinical \& Translational Oncology 2006; 8: 616-617.

15. Ambrogi V, Nofroni I, Tonini G, Mineo TC. Skin metastases in lung cancer: analysis of a 10-year experience. Oncology Reports 2001; 8: 57-61.

16. Pantarotto M, Lombo L, Pereira H, et al. Cutaneous metastasis as the initial manifestation of lung adenocarcinoma. Jornal Brasileiro de Pneumologia 2012; 37: 556-9.

17. Kamble R, Kumar L, Kochupillai V, et al. Cutaneous metastases of lung cancer. Postgrad Medical Journal 1995; 71: 741743 .

18. Barbetakis N, Samanidis G, Paliouras D, et al. Facial skin metastasis due to small-cell lung cancer: a case report. Journal of Medical Case Reports 2009, 3:32-34.

19. Maeda J, Miyake M, Tokita K, et al. Small Cell Lung Cancer with Extensive Cutaneous and Gastric Metastases. Internal Medicine 1992, 11:1325-1328.

\section{REFERENCES}

1. Wright $\mathrm{T}$. Cutaneous manifestations of malignancy. Current Opinion in Pediatrics. 2011; 23: 407-411.

2. Asher R, Hollowood K. Primary cutaneous lymphoma: An overview based on the WHO-EORTC classification. Diagnostic Histopathology 2010; $16: 168-181$.

3. Intracaso C, Kantor J, Porter D, et al. Cutaneous Hodgkin's disease. Journal of American Academy of Dermatology 2008; 58: 295-298. 